\title{
The Selective Inhibition of Growth Cone Extension by Specific Neurites in Culture
}

\author{
Josef P. Kapfhammer, Barbara E. Grunewald, and Jonathan A. Raper \\ Max-Planck-Institut für Entwicklungsbiologie, 7400 Tübingen, Federal Republic of Germany
}

\begin{abstract}
We have found that neurites from embryonic chick retinal and sympathetic explants do not mix in culture, while retinal neurites mix with retinal neurites, and sympathetic neurites mix with sympathetic neurites. These results confirm those obtained with embryonic rat tissues by Bray et al. (1980). We have also used video time-lapse techniques to examine the behavior of individual retinal and sympathetic growth cones as they attempt to cross retinal and sympathetic neurites. We have found that (1) retinal growth cones cross retinal neurites without delay, (2) retinal growth cones usually retract from sympathetic neurites but often cross them on a second advance, (3) sympathetic growth cones repeatedly retract from retinal neurites, rarely managing to cross them at all, and (4) sympathetic growth cones cross sympathetic neurites but are sometimes delayed.

Our results indicate that retinal and sympathetic growth cones can distinguish between specific labels associated with retinal and sympathetic neurites. They also suggest that active avoidance could play an important role in growth cone navigation.
\end{abstract}

Growth cones must use information gleaned from their surrounding environment to extend upon their correct pathways towards their appropriate targets. The distributions of a great variety of environmental factors could either direct growth cones along, or force them into, their correct pathways. Relevant cues might include extracellular matrix components permissive for neurite outgrowth, e.g., fibronectin (Akers et al., 1981) or laminin (Hammarback et al., 1985; Manthorpe et al., 1983); soluble factors that affect growth cone orientation, e.g., NGF (Gundersen and Barrett, 1980); soluble factors that inhibit the motility of specific growth cones, e.g., 5-HT (Haydon et al., 1984); electrical fields (e.g., Patel and Poo, 1984); molecules on the surfaces of non-neuronal cells (Fallon, 1985; Noble et al., 1984; Silver and Rutishauser, 1984); the mechanical constraints imposed by extracellular channels (Silver and Sidman, 1980; Singer et al., 1979); or impenetrable tissue barriers (Tosney and Landmesser, 1984). Another important environmental feature that may help to guide growth cones are the axons they contact during their extension in the developing embryo.

There is evidence from both invertebrates and vertebrates that growth cones distinguish between and grow upon specific axons. In the CNS of the grasshopper, each of the growth cones in a family of identified neurons grows in direct contact with a specific, reproducible sequence of axons (Bastiani et al., 1984; Raper et al., 1983). These findings suggested the "labeled path-

\footnotetext{
Received Oct. 2, 1985; revised Jan. 20, 1986; accepted Jan. 21, 1986.

We thank Susannah Chang and Friedrich Bonhoeffer for many helpful discussions and S.C., Rupert Hallmann, and Donald Newgreen for their criticism of the manuscript. We are especially indebted to F.B. for his advice during the video time-lapse study. This work was supported by the Max-Planck-Gesellschaft. J.P.K. received a stipend from the Studienstiftung des deutschen Volkes.

Correspondence should be addressed to Jonathan A. Raper, MPI Entwicklungsbiologie, Spemannstr. 35, 7400 Tübingen, Federal Republic of Germany.

Copyright (C) 1986 Society for Neuroscience $0270-6474 / 86 / 092527-08 \$ 02.00 / 0$
}

ways" hypothesis (Goodman et al., 1982), namely, that early differentiating neurons construct a scaffolding of labeled axonal pathways that subsequent growth cones choose between and elongate upon. The deletion of specific axons in selected pathways has been shown to interfere with the ability of subsequent growth cones to navigate properly in the grasshopper CNS (du Lac and Goodman, 1984; Raper et al., 1984).

An example of vertebrate growth cones distinguishing between and preferring to grow upon specific axonal substrates has been described for explanted chick retinal tissues (Bonhoeffer and Huf, 1985). When temporal retinal axons are given the opportunity in a simple choice experiment of extending upon either temporal or nasal axons, they always grow upon other temporal fibers. Nasal growth cones show no preference between nasal and temporal axons.

Bray et al. (1980) have described another vertebrate tissue culture system in which neurite-neurite recognition appears to occur. They explanted various combinations of embryonic rat retinal and superior cervical ganglion (SCG) tissues next to each other on a collagen substrate. The neurites from 2 adjacent retinal explants were found to intermix, as were the neurites from 2 adjacent SCGs. However, when an SCG was placed next to a retinal explant, the neurites from each explant were confined to separate territories.

We have found that embryonic chicken sympathetic and retinal tissues do not mix when grown on a laminin substrate. In addition, we describe the behavior of individual sympathetic and retinal growth cones as they contact sympathetic and retinal neurites. Our results support the idea that growth cones can distinguish between differentially labeled axons, raising the possibility that some of the labels involved have distinctly negative effects on growth cone extension.

\section{Materials and Methods}

\section{Explants}

Retinal explants were made by the procedure described in Halfter et al. (1983). Briefly, embryonic day 6 (E6) neural retinas were spread on concanavalin A (Con-A)-coated nitrocellulose filters and cut into 300$\mu \mathrm{m}$-wide strips with a tissue chopper (Bachofer). The strips were cut parallel to the optic fissure. We used tissue immediately adjacent to and from both the nasal and temporal sides of the fissure. Strips were then explanted ganglion cell layer down. Single E8 or E9 sympathetic ganglia were taken from the lumbosacral region. Pairs of explants were placed 2-3 $\mathrm{mm}$ apart on laminin-coated glass coverslips (see below). The explants were temporarily held in place with weighted dialysis membranes for 5-30 min, after which they were firmly attached to the substratum and the membranes removed. Usually, 3 coverslips were cultured for 1-7 d in 5-cm-diameter petri dishes containing $5 \mathrm{ml}$ of medium (see below) in $5 \% \mathrm{CO}_{2}$ at $37^{\circ} \mathrm{C}$.

\section{Substrate}

The explants were grown on laminin-coated glass coverslips (Bonhoeffer and Huf, 1985). Coverslips $9 \mathrm{~mm}$ in diameter were first cleaned in boiling nitric acid, washed, and then incubated with 10\% 3-(triethoxysil- 
yl)propylamine in dimethylsulfoxide (DMSO) at approximately $37^{\circ} \mathrm{C}$ for 2-4 hr. They were washed in DMSO, then distilled water, sterilized under a UV lamp, and stored until needed. Just before use, 2 coverslips were coated simultaneously with laminin by sandwiching between them a $25 \mu$ d drop of laminin solution [ $20 \mu \mathrm{g}$ laminin (BRL) $/ \mathrm{ml}$ of calciumfree Hank's balanced salt solution]. After $1-2 \mathrm{hr}$ at $37^{\circ} \mathrm{C}$, they were washed in Hank's and were ready for use.

\section{Medium}

All dissections were performed in calcium- and magnesium-free Hank's. All cultures were grown in F12 medium supplemented as in Bottenstein et al. (1980), plus $2 \mathrm{~mm}$ glutamine, $100 \mathrm{U} / \mathrm{ml}$ penicillin, $100 \mathrm{ng} / \mathrm{ml}$ streptomycin, $20 \mathrm{ng} / \mathrm{ml} 7 \mathrm{~S}$ NGF (Sigma), $10^{-5} \mathrm{M}$ cytosine arabino-furanoside (Sigma), $10^{-5} \mathrm{M}$ S-Fluorodeoxy uridine (Sigma), $5 \%$ fetal calf serum (Serva), and 5\% chicken serum (Gibco).

\section{Time-lapse video recordings}

Cultures were maintained in culture dishes that had thin, transparent, plastic film bottoms (hydrophilic Petriperm). These dishes were placed on the stage of an inverted microscope (Zeiss IM 35) kept in a $37^{\circ} \mathrm{C}$ warm room. A silicon ring with a glass cover was placed over the culture dish, and humidified air containing $5 \% \mathrm{CO}_{2}$ was delivered into this chamber under slight pressure. A TV camera (National WV-261) was fitted to the microscope and connected to a time-lapse video recorder (National NV-8030). Real time was simultaneously recorded on the video image using a time-date generator (National WJ-800). Single images were recorded every 2.5 or $5 \mathrm{sec}$, yielding acceleration factors of $125 x$ or $250 \times$, respectively. The recordings were viewed on a video monitor (National WV-5400).

\section{Analysis of time-lapse video recordings}

All time-lapse recordings were made when the cultures were between 20 and $72 \mathrm{hr}$ old. Fields of view $(220 \times 350$ or $340 \times 550 \mu \mathrm{m})$ were chosen in zones of confrontation that were not too crowded, and in which the axons and growth cones involved could be traced back to their respective explants. The video tapes were screened for instances in which single growth cones approached axons or small fascicles at roughly orthogonal angles between $60^{\circ}$ and $120^{\circ}$. Recordings in which the identity of any participant was ambiguous or that included nonneuronal cells were discarded. Also discarded werc recordings in which frequent spontaneous growth cone retractions were observed (usually an indication that the substrate was poor). When an acceptable confrontation was found, a transparent plastic sheet was layered over the monitor, the necessary axons were traced onto the sheet, and the positions of relevant growth cones recorded on the sheet for every $5 \mathrm{~min}$ of real elapsed time.

It should be noted that these methods may have biased our data to reflect the behavior of faster-growing growth cones. This is because we limited our data collection to the first growth cones to meet opposing axons and to growth cones that did not undergo frequent spontaneous retractions. It should also be noted that we observed very little fasciculation. This is probably because we analyzed only growth cone-neurite encounters with roughly orthogonal angles of approach and because the laminin substrate we used supports neurite outgrowth approximately as well as the surfaces of other axons.

\section{Results}

\section{Confrontations between pairs of explants}

When retinal and sympathetic tissues are taken from embryonic chicks and are cultured together on laminin-coated glass for 4 $\mathrm{d}$ or more, their axons do not mix freely. Instead, they form distinct and apparently separate axonal territories (Fig. 1A). These axonal territories are characterized by the crowding of axons on the sides of the explants that confront each other (Fig. $1 A$, arrows). This probably occurs as axons are deflected away from what would be their unperturbed courses. Retinal and sympathetic territories are usually, but not always, separated by a zone in which a relatively small number of axons crosses from one territory to the other (between arrows in Fig. $1 A$ and arrows in Fig. $1 B$ ). Those sympathetic axons that do cross into retinal territory are generally gathered into thick fascicles (Fig. $1 B$, arrowheads). This is in contrast to their usual tendency to split into ever finer fascicles and single axons as they grow away from a solitary sympathetic ganglion. As reported by Bray et al. (1980), these invading sympathetic fascicles do not mix freely with, and are often observed to be at slightly different focal planes from, the retinal axons. A nearly axon-free gap is also observed around invading sympathetic fascicles, although here, as in other regions of the axon-free zone, some very fine processes traverse the gap at right angles to the lines of confrontation.

When either 2 retinal (Fig. $2 A$ ) or 2 sympathetic (Fig. $2 B$ ) explants confront each other, their neurites appear to mix freely. In some sympathetic-sympathetic confrontations, neurites mix but are slightly deflected from what would be their unperturbed, radially symmetric trajectory if growing from a single explant (not shown).

\section{Time-lapse recordings}

The videotapes used in this study were made at relatively low magnifications so that we could first examine the overall unperturbed behavior of extending individual growth cones, and then, on the same video recording, watch the same growth cones as they interacted with neurites in their path. The images obtained were of sufficient quality to accurately locate and identify growth cones, neurites, and non-neuronal cells, but their resolution was not sufficient to study the detailed morphology of growth cones (Fig. 3).

Data were collected as described in Materials and Methods and then converted to plots that related the relative positions of a growth cone and the neurite it approached as a function of time. To do this we first drew a straight line through the neurite. This served as an approximate description of the neurite's overall orientation (dashed line in Fig. $4 A$ ). Then, for each time point, the distance between the center of the growth cone and the neurite was measured perpendicular to this line (dashed line with arrowhead in Fig. 4A). Distances measured before the crossing were defined as positive, and those after crossing as negative. The plot resulting from the cxample in Figure $4 A$ is shown in Figure $4 B$. The curve represents growth cone displacement relative to the neurite as a function of time, and the slope of the line represents the velocity at which the growth cone approaches the neurite. We have found it useful to define several other parameters that can be measured from these plots (Fig. $4 C)$ :

1. "Crossing" signifies that the growth cone crosses the neurite and continues to translocate away from it.

2. "Retraction" ( $\mathrm{R}$ in Fig. $4 C$ ) signifies that during a growth cone's approach towards a neurite there is a phase in which the distance between them increases, rather than steadily decreasing.

3. "Delay" (D in Fig. 4C) is the interval between the projected time when the unperturbed growth cone would cross the neurite and the time at which it actually does cross the neurite. In the absence of crossing, the delay has an infinite value.

\section{Unperlurbed growth cones}

On a uniform laminin substrate, retinal growth cones grow at a relatively steady rate, with only very rare spontaneous retractions. Branching occurs but is uncommon. Retinal growth cones in our preparations typically extend between 80 and $250 \mu \mathrm{m} /$ hr. Sympathetic growth cones do not grow as well as retinal growth cones in the culture conditions we used. Spontaneous retractions and branching occur more often with sympathetic than with retinal neurites but are still infrequent. Sympathetic growth cones typically extend between 60 and $200 \mu \mathrm{m} / \mathrm{hr}$.

\section{Retinal growth cones meeting retinal axons}

Retinal growth cones usually cross retinal neurites without delay or any sign of retraction (Fig. $5, A 1-A 3$ ). The growth cones 

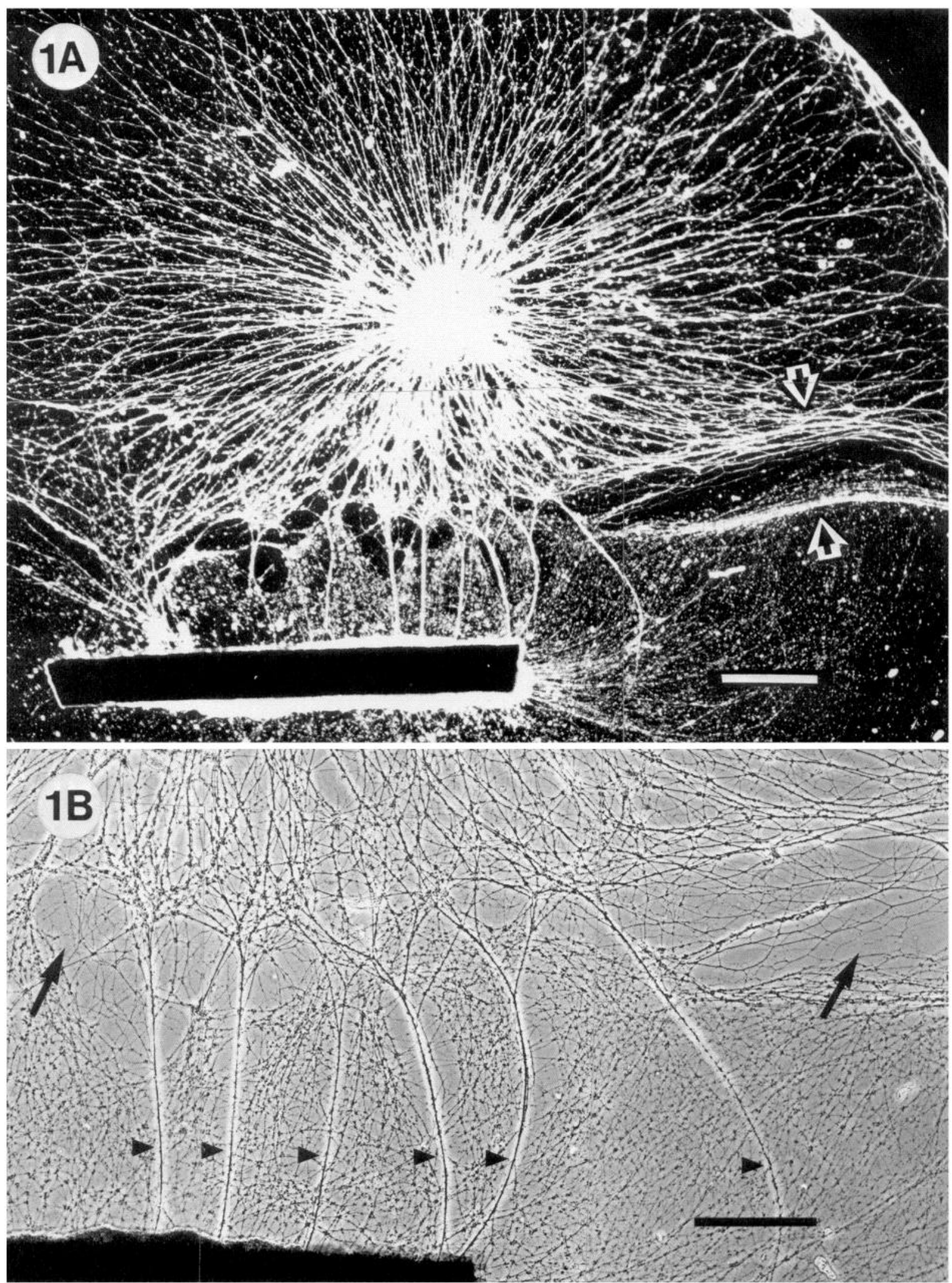

Figure 1. A, Neurites from a sympathetic explant (top) confront neurites from a retinal explant (bottom strip). Deflected sympathetic and retinal neurites are separated by a nearly axon-free gap (arrows). Calibration, $600 \mu \mathrm{m}$. B , Higher-magnification view of the same culture in the region directly between the explants. Two nearly axon-free gaps between sympathetic (top) and retinal (bottom) territories are indicated by arrows. Sympathetic neurites are gathered into tight bundles as they enter retinal territory (arrowheads). Calibration, $300 \mu \mathrm{m}$. 

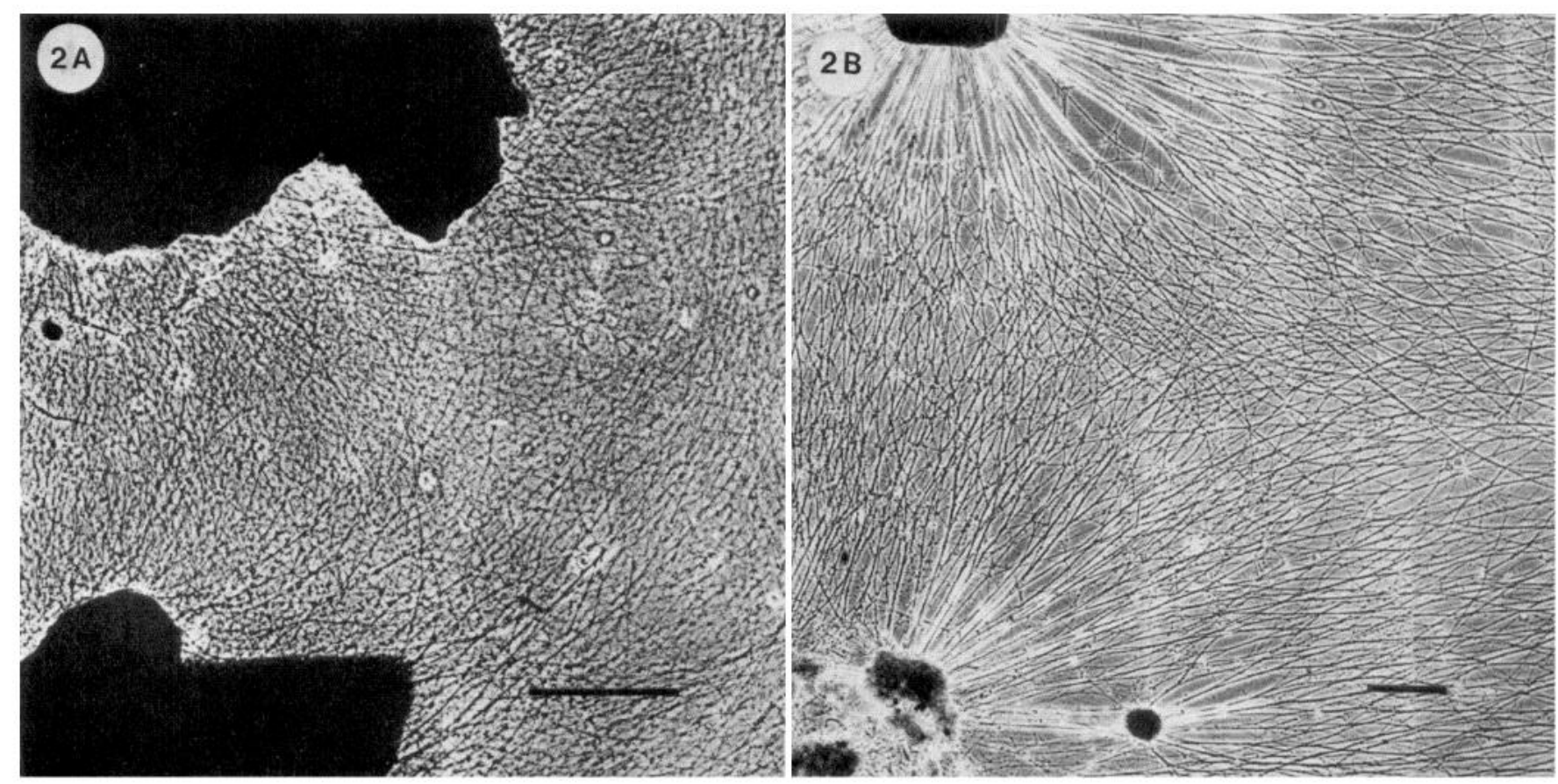

Figure 2. A, Neurites from 2 retinal explants (top and bottom) confront each other. B, Neurites from 2 sympathetic explants (top and bottom) confront each other. Calibrations, $300 \mu \mathrm{m}$.

advance as if they were growing on a normal laminin substrate, ignoring the retinal neurites as they cross them. Rarely do growth cones slow down and remain in contact with retinal neurites before crossing them (Fig. 5, A4, A5).

\section{Retinal growth cones meeting sympathetic axons}

Retinal growth cones appear to cross sympathetic neurites with considerable difficulty. Typically, they advance at a steady pace until they touch a sympathetic axon, but they then retract away from it. These retinal growth cones usually begin to advance again after a recovery period of between 10 and $60 \mathrm{~min}$. A growth cone may make a successful crossing on its second attempt (Fig. $5 B 1$ ), or the retraction cycle can be repeated several times before a successful crossing occurs (Fig. 5B2). In a minority of encounters, retinal growth cones never cross sympathetic neurites (e.g., Fig. 5B3). In a still smaller class of encounters, retinal growth cones cross sympathetic neurites without retraction or delay (e.g., Fig. 5B4).

\section{Sympathetic growth cones meeting sympathetic axons}

Sympathetic growth cones almost always cross sympathetic neurites, although with more difficulty than is seen in retinal-retinal encounters. Some sympathetic growth cones cross with little or no delay (Fig. 5, C1, C2). Others are delayed without retracting (Fig. $5 C 3$ ), and some even retract before ultimately crossing (Fig. 5C4).

\section{Sympathetic growth cones meeting retinal axons}

Sympathetic growth cones usually do not cross retinal neurites. They retract upon contact, usually recover, and advance once again. This retraction cycle can be repeated several times (Fig. $5, D 1, D 2)$. In some cases, a sympathetic growth cone becomes quiescent upon contact, neither retracting nor crossing (Fig. 5D3). In rare cases, sympathetic growth cones cross retinal neurites with only a brief delay (Fig. 5D4).

\section{Quantitative comparisons}

All these data are summarized in Table 1 and Figure 6. For the purposes of Table 1, "retraction" signifies that the growth cone withdrew at least $7 \mu \mathrm{m}$, a distance that is approximately one growth cone diameter. If appropriate subsets of the data in this table are arranged in the form of standard $2 \times 2$ statistical comparisons, it is possible to estimate the probability that a given type of growth cone behaves identically when it meets retinal, as compared to sympathetic, neurites:

1. The probability that retinal growth cones cross retinal and sympathetic neurites with equal frequency is $p<0.001$.

2 . The probability that they retract with equal frequency is $p<0.001$.

3. The probability that sympathetic growth cones cross retinal and sympathetic neurites with equal frequency is $p<0.001$.

4. The probability that they retract with equal frequency is $p<0.001$.

Figure 6 presents histograms showing the frequency of delays for each type of encounter. Note that retinal growth cones cross retinal neurites with less delay than sympathetic neurites, and that sympathetic growth cones cross sympathetic neurites with less delay than retinal neurites. This effect is superimposed upon a general tendency for retinal growth cones to cross neurites with a shorter delay than sympathetic growth cones.

\section{Discussion}

We have confirmed the finding by Bray et al. (1980) that neurites extending from cultured retinal explants prefer not to mix with neurites extending from sympathetic explants, while retinal neurites mix with retinal neurites, and sympathetic neurites mix with sympathetic neurites. This is a robust phenomenon since our results were obtained, in comparison to those of Bray et al., using chick instead of rat tissues, culturing on a 2-dimensional laminin substrate instead of in a 3-dimensional collagen network, and with a different culture medium. It is therefore unlikely that the specific tendency for retinal and sympathetic neurites to remain separate is an artifact of the particular conditions in which they are cultured.

It is important to note that non-neuronal cells do not play a role in this phenomenon. Neuronal processes extend much more rapidly on laminin than do non-neuronal cells, so that, on this substrate, opposing neurites from adjacent explants meet before 


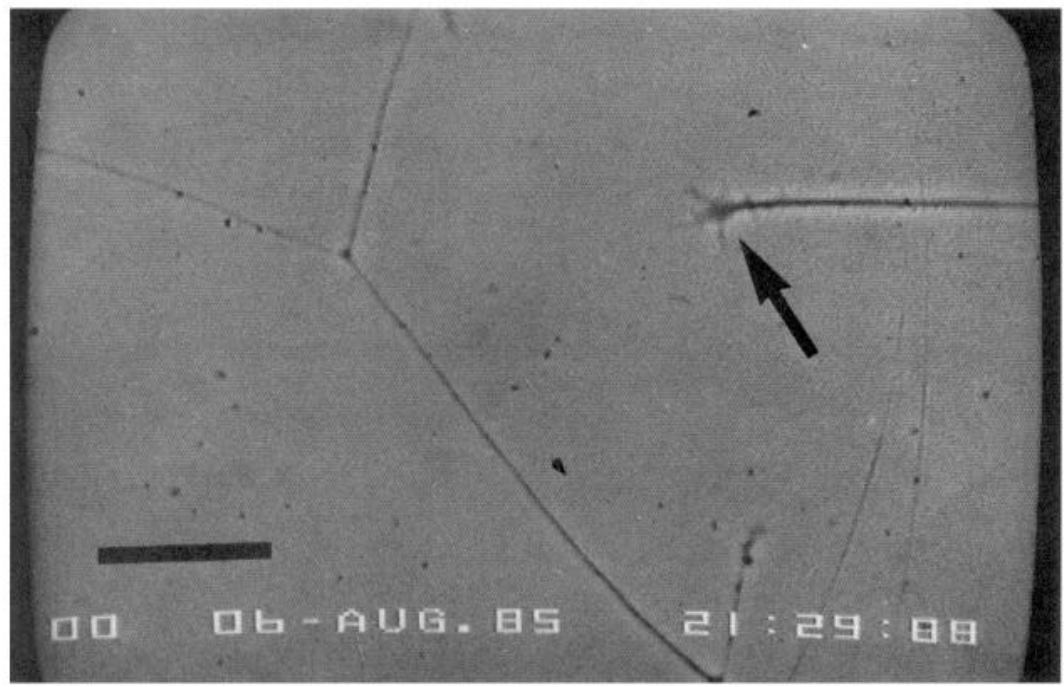

Figure 3. Video monitor display showing a sympathetic growth cone (arrow) growing toward a neurite. Real time and date are indicated on the screen.

the arrival of non-neuronal cells. In our cultures, non-neuronal cells were further selected against with antimitotic agents. Almost no non-neuronal cells were observed in the zone where neurites confronted each other. More importantly, our timelapse studies rigorously excluded all non-neuronal cells.

We have observed and quantified the behavior of individual identified growth cones as they encounter identified neurites. It is difficult to predict the outcome of any single growth coneneurite encounter with certainty or to describe all outcomes succinctly. Perhaps this is because the behavior of growth cones has a stochastic component or because sympathetic and/or retinal cells are not homogenous in their properties. However, it is possible to make valid statements concerning classes of encounters. (1) As a general rule, sympathetic growth cones are

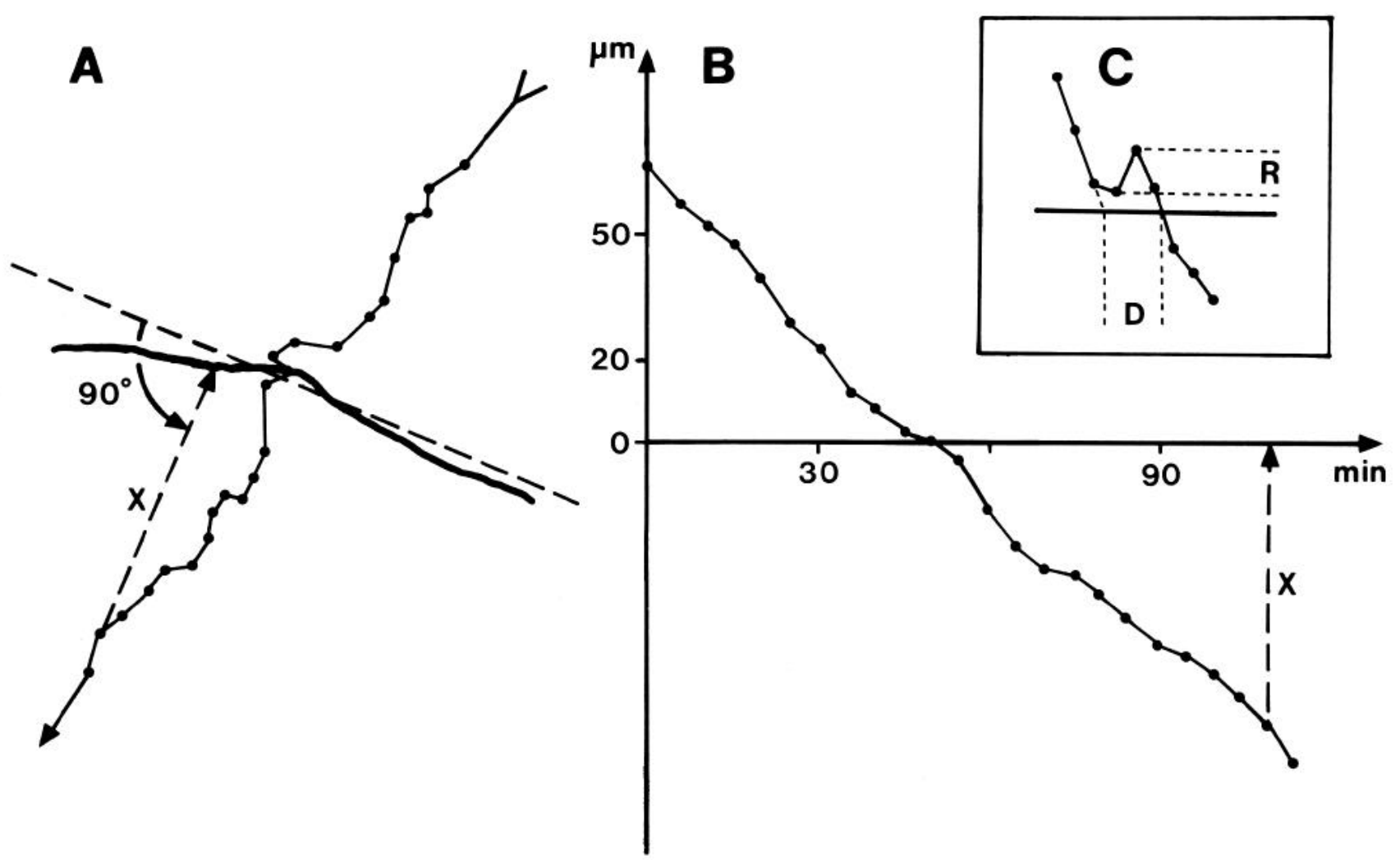

Figure 4. Data analysis of video recordings. A, Tracing from the video screen of a sympathetic growth cone crossing a sympathetic neurite. Black dots indicate the positions of the growth cone center at $5 \mathrm{~min}$ intervals. The neurite is indicated by the thick solid line. Distances between the growth cone and the neurite were measured perpendicular to the dashed line. $B$, Plot of the distances between growth cone and neurite vs time from $A$. Black dots indicate distances at $5 \mathrm{~min}$ intervals. The position of the neurite coincides with the $x$-axis. The distance $X$ is the same in $A$ and $B . C$. Definition of terms: delay $(D)$ is the difference between the times when the unperturbed growth cone should have crossed the neurite (dashed line) and when it actually did (dashed line). Retraction $(R)$ is the difference between the distance separating the growth cone and neurite at their point of closest approach (dashed line), and the distance separating them just prior to the growth cone's subsequent readvance (dashed line). 


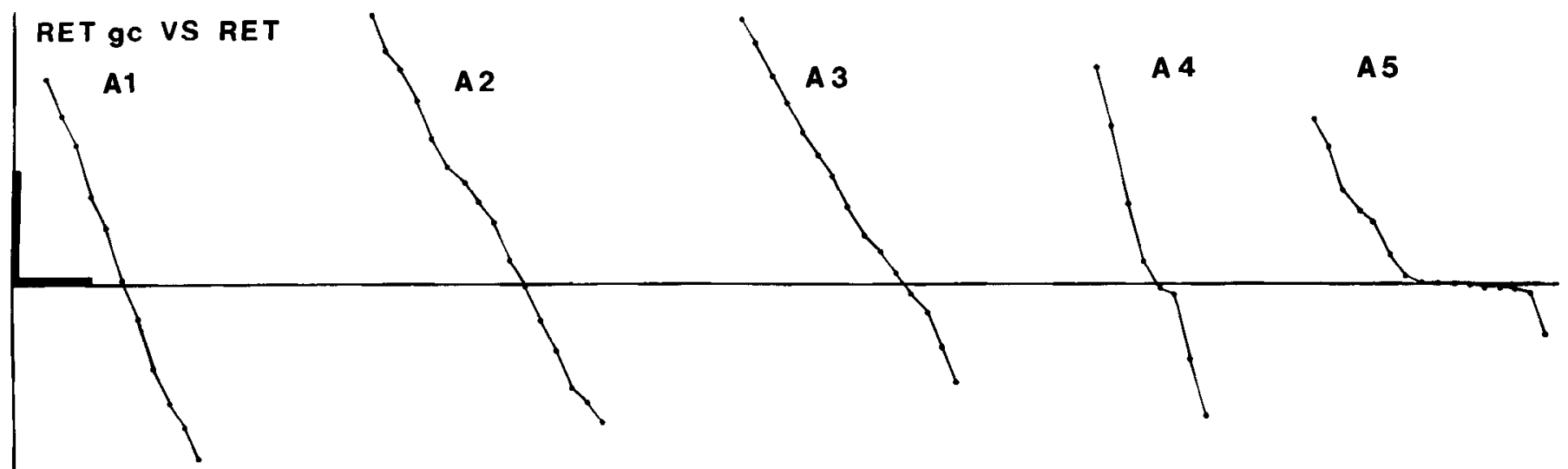

RET gC VS SYMP

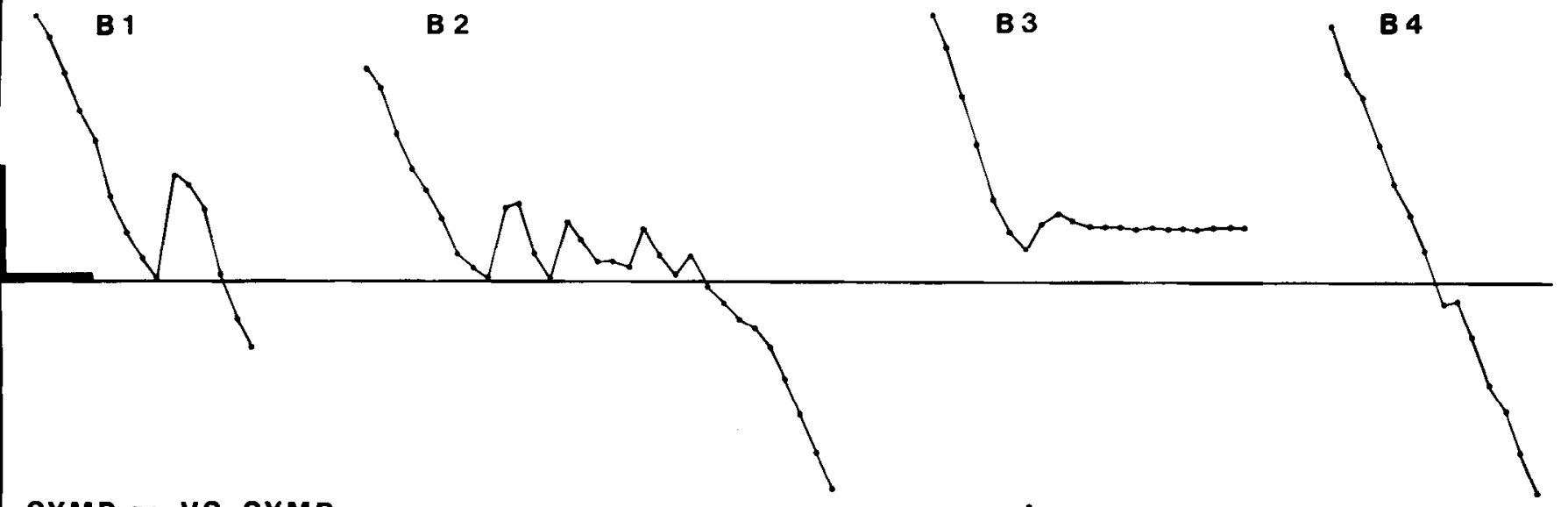

SYMP gc VS SYMP

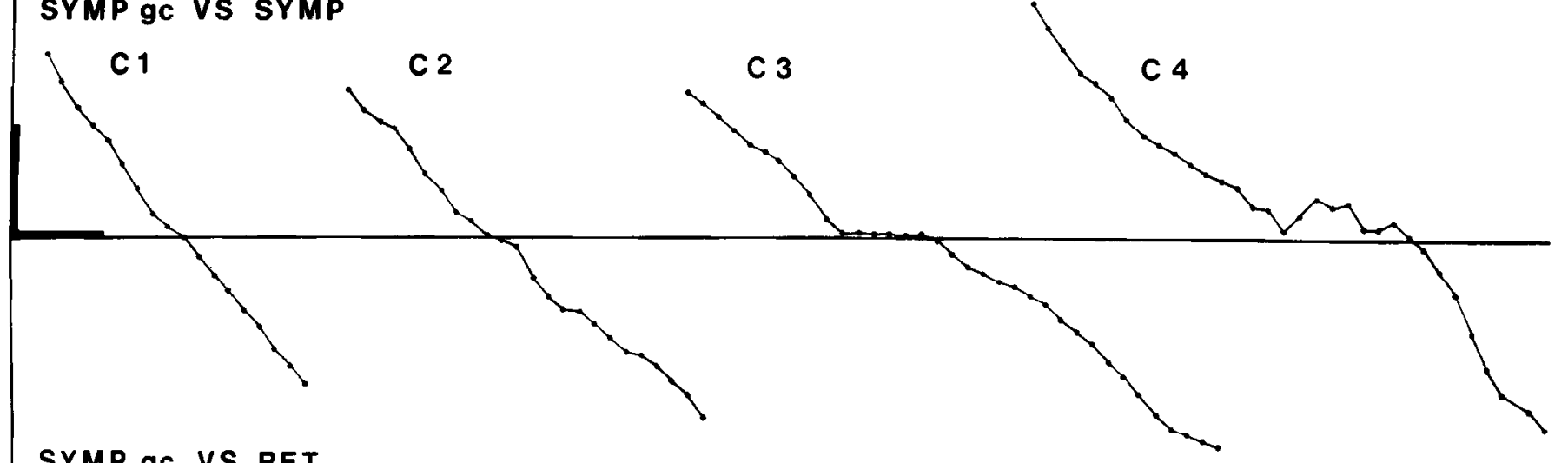

SYMP gC VS RET

D 1

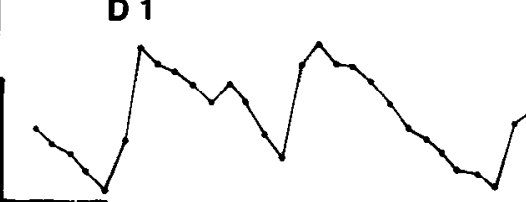

D 2

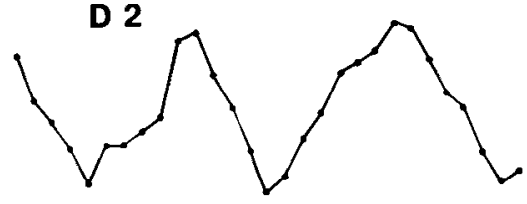

D 3

D 4

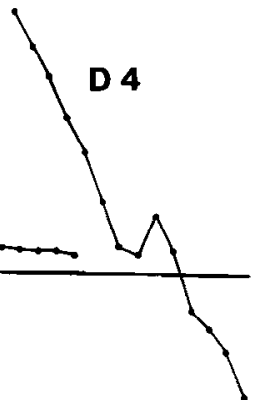

Figure 5. A selection of encounters between growth cones and neurites. The method described in Figure 4 was used to plot the distances between the center of growth cones and the neurites they approached. $A 1-A 5$, Retinal growth cones meeting retinal neurites. B1-B4, Retinal growth cones meeting sympathetic neurites. C1-C4, Sympathetic growth cones meeting sympathetic neurites. DI-D4, Sympathetic growth cones meeting retinal neurites. Panels towards the left tend to represent typical cases, while those towards the right tend to represent exceptions. See text for details. Calibrations (thickened portions) of $X$-axis: $30 \mathrm{~min}$; $Y$-axis: $50 \mu \mathrm{m}$. 
Table 1. Summary of all growth cone-neurite interactions analyzed by time-lapse video recordings

\begin{tabular}{lcrrrrrr} 
Interaction & $\mathrm{N}$ & \multicolumn{1}{c}{$\mathrm{R}$} & \multicolumn{1}{c}{ No R } & $\% \mathrm{R}$ & $\mathrm{X}$ & No X \%X \\
\hline Ret gc vs ret & 44 & 1 & 43 & 2 & 44 & 0 & 100 \\
Ret gc vs symp & 38 & 30 & 8 & 79 & 27 & 11 & 68 \\
Symp gc vs symp & 29 & 8 & 21 & 28 & 27 & 2 & 93 \\
Symp gc vs ret & 24 & 23 & 1 & 96 & 7 & 17 & 29
\end{tabular}

Each row summarizes the data for the type of growth cone-neurite interaction indicated at left. $N$ is the number of cases analyzed. $\mathrm{R}$ signifies at least one retraction of $7 \mu \mathrm{m}$ or more, No $R$ signifies no retraction, and $\% R$ is the percentage retracting. $X$ signifies crossing, No $X$ signifies no crossing, and $\% X$ is the percentage crossing. Since retracting growth cones can ultimately cross, the 2 measures are not identical.

more likely to be delayed by neurites in their path than are retinal growth cones, and (2) sympathetic growth cones are delayed less by sympathetic neurites than they are by retinal neurites, while retinal growth cones are delayed more by sympathetic neurites than they are by retinal neurites.

Several observations suggest that sympathetic growth cones are less robust in our cultures than are retinal growth cones and, for this reason, are at a disadvantage in crossing neurites in their path. We find that retinal growth cones grow faster, cross retinal axons without delay, and very often manage to cross sympathetic neurites. This is in contrast to sympathetic neurites, which grow more slowly, rarely cross retinal neurites, and are sometimes delayed even by sympathetic neurites. Although our sympathetic growth cones are slightly delayed by sympathetic neurites, our results differ from those of Dunn (1971), who reported that chick dorsal root ganglion (DRG) growth cones display a "contact inhibition of extension" when they meet DRG neurites grown in plasma clots. On the whole, our findings are more similar to those of Wessells et al. (1980), who found, for example, that chick ciliary growth cones cross ciliary neurites without retracting.

Our major finding is that retinal growth cones are delayed less by retinal neurites than by sympathetic neurites and, conversely, that sympathetic growth cones are delayed more by retinal neurites than by sympathetic neurites. This cannot be explained by any scheme that assumes that neurites act as passive mechanical barriers. If, for example, one neurite type is more obstructive to growth cone advancement than another, then both retinal and sympathetic growth cones would be most delayed by that one type of neurite. Nor does it help to invoke the greater robustness of retinal growth cones as compared to sympathetic growth cones, since sympathetic growth cones would then suffer an additional delay that would not depend upon the type of neurite involved. This would not help to explain why retinal and sympathetic growth cones are most delayed by different types of neurites.

Our results can only be explained if there exists at least one specific difference between retinal and sympathetic neurites that allows growth cones to distinguish between them. There must also be at least one additional complementary difference between retinal and sympathetic growth cones that allows them to respond differently to identical cues.

The difficulties experienced by retinal growth cones attempting to cross sympathetic neurites, or, even more strikingly, by sympathetic growth cones attempting to cross retinal axons, can most easily be characterized as involving the withdrawal of the growth cones. In a subsequent paper (Kapfhammer and Raper, in press) we show that withdrawal is initiated by a collapse in growth cone morphology resembling type I contact-mediated inhibition (Heaysman, 1978). After a brief pause, a new growth cone forms and again advances. This retraction-advance cycle can be repeated several times. It is therefore our impression

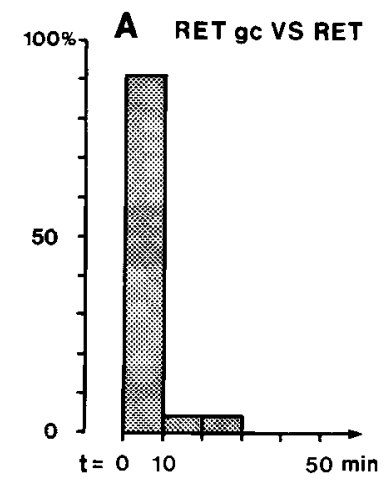

B RET ge VS SYMP
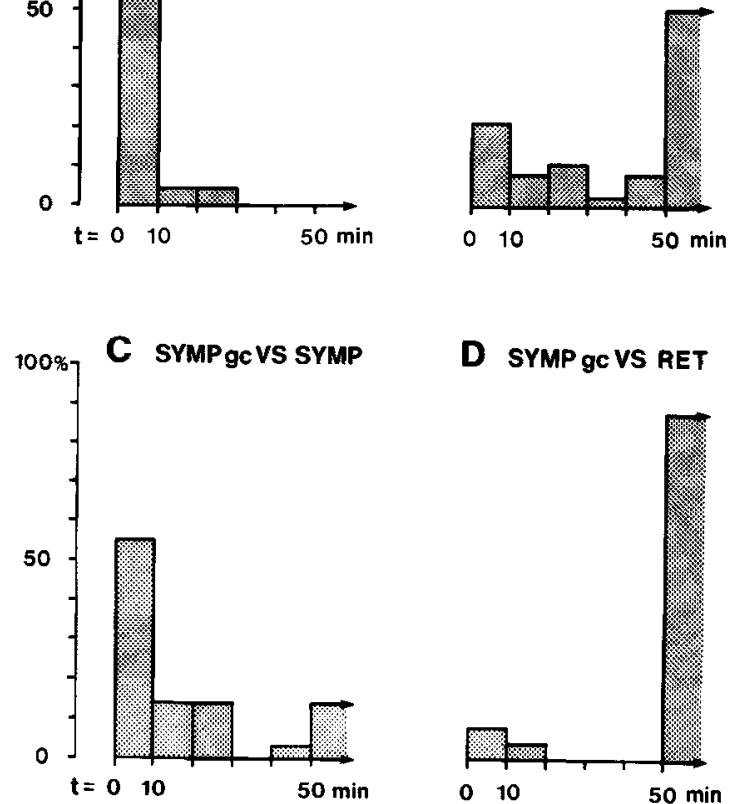

Figure 6. Frequency histograms of the delay times for growth cones crossing neurites. Column heights represent the percentage of delays that fell into a particular $10 \mathrm{~min}$ interval. The last column of each histogram represents all delay times greater than $50 \mathrm{~min}$. Delay is defined in Figure $4 C$. $A$, Retinal growth cones meeting retinal neurites. $B$, Retinal growth cones meeting sympathetic neurites. $C$. Sympathetic growth cones meeting sympathetic neurites. $D$, Sympathetic growth cones meeting retinal neurites.

that when a growth cone meets an incompatible neurite there is an immediate negative effect upon growth cone activity and locomotion. Withdrawal from the neurite effects a recovery of growth cone function within tens of minutes. It is not uncommon for a second or third advance to result in a successful crossing. This raises the possibility of habituation in some component of the recognition or retraction processes.

The experiments cited in the introduction suggest that there are specific labels on axons that make them more or less attractive to particular growth cones. In theory, this could occur if molecules promoting specific positive interactions of varying degrees are associated with particular axons and growth cones in varying amounts. In this way a hierarchy of preferences could be constructed from wholly positive interactions, a scheme like that proposed by Steinberg (1970) for differential cell adhesion. In explaining growth cone navigation, this kind of model would require that extending growth cones seek out ever more attractive substrates in order to stay "on track." For this reason, neurite initiation might have to occur in relatively inhospitable environments, and the choices available to growth cones would become increasingly constrained as they advance.

One way around these limitations would be for growth cones to change their preferences over time. Another solution might be provided by the intermixing of environmental cues with both positive and negative effects on the motility of specific growth cones. Finally, growth-permissive substances might be generally distributed, while specific growth-inhibiting substances would be found only in particular localized regions. This would allow 
growth cones to extend along highly defined, specific pathways without ever having to grow on suboptimal substrates.

Our results support the idea that growth cones recognize and respond to labels associated with specific axons. They also suggest that some of these labels have distinctly negative effects upon growth cone elongation.

\section{References}

Akers, R. M., D. F. Mosher, and J. E. Lilien (1981) Promotion of retinal neurite outgrowth by substratum-bound fibronectin. Dev. Biol. 86: 179-188.

Bastiani, M. J., J. A. Raper, and C. S. Goodman (1984) Pathfinding by neuronal growth cones in grasshopper embryos: III. Selective affinity of the $G$ growth cone for the $P$ cells within the $A / P$ fascicle. $J$. Neurosci. 4: 2311-2328.

Bonhoeffer, F., and J. Huf (1985) Position-dependent properties of retinal axons and their growth cones. Nature 315: 409-410.

Bottenstein, J. E., S. D. Skaper, S. S. Varon, and G. H. Sato (1980) Selective survival of neurons from chick embryo sensory ganglionic dissociates utilizing serum-free supplemented medium. Exp. Cell Res. 125: 183-190.

Bray, D., P. Wood, and R. P. Bunge (1980) Selective fasciculation of nerve fibers in culture. Exp. Cell Res. 130: 241-250.

du Lac, S., and C. S. Goodman (1984) Selective fasciculation in the grasshopper embryo: Experimental test of the labelled pathways hypothesis. Soc. Neurosci. Abstr. 10: 140.

Dunn, G. A. (1971) Mutual contact inhibition of extension of chick sensory nerve fibers in vitro. J. Comp. Neurol. 143: 491-508.

Fallon, J. R. (1985) Preferential outgrowth of central nervous system neurites on astrocytes and Schwann cells as compared with nonglial cells in vitro. J. Cell Biol. 100: 198-207.

Goodman, C. S., J. A. Raper, R. Ho, and S. Chang (1982) Pathfinding by neuronal growth cones during grasshopper embryogenesis. Symp. Soc. Dev. Biol. 40: 275-316.

Gundersen, R. W., and J. N. Barrett (1980) Characterization of the turning response of dorsal root neurites toward nerve growth factor. J. Cell Biol. 87: 546-554.

Halfter, W., D. F. Newgreen, J. Sauter, and U. Schwarz (1983) Oriented axon outgrowth from avian embryonic retinae in culture. Dev. Biol. 95: 56-64.

Hammarback, J. A., S. L. Palm, L. T, Furcht, and P. C. Letourneau (1985) Guidance of neurite outgrowth by pathways of substratumadsorbed laminin. J. Neurosci. Res. 13: 213-220.
Haydon, P. G., D. B. McCobb, and S. B. Kater (1984) Serotonin selectively inhibits growth cone motility and synaptogenesis of specific identified neurons. Science 226: 561-564.

Heaysman, J. E. M. (1978) Contact inhibition of locomotion: A reappraisal. Int. Rev. Cytol. 55: 49-66.

Kapfhammer, J. P., and J. A. Raper (in press) The collapse of growth cone structure on contact with specific neurites in culture. J. Neurosci.

Manthorpe, M., E. Engvall, E. Rouslahti, F. M. Longo, G. E. Davis, and $S$. Varon (1983) Laminin promotes neuritic regeneration from cultured peripheral and central neurons. J. Cell Biol. 97: 1882-1890.

Noble, M., J. Fok-Seang, and J. Cohen (1984) Glia are a unique substrate for the in vitro growth of central nervous system neurons. J. Neurosci. 4: 1892-1903.

Patel, M. B., and M.-M. Poo (1984) Perturbation of the direction of neurite growth by pulsed and focal electric fields. J. Neurosci. 4: 29392947.

Raper, J. A., M. J. Bastiani, and C. S. Goodman (1983) Pathfinding by ncuronal growth cones in grasshopper embryos: II. Selective fasciculation onto specific axonal pathways. J. Neurosci. 3: 31-41.

Raper, J. A., M. J. Bastiani, and C. S. Goodman (1984) Pathfinding by neuronal growth cones in grasshopper embryos: IV. The effects of ablating the $A$ and $P$ axons upon the behavior of the $G$ growth cone. J. Neurosci. 4: 2329-2345.

Silver, J., and U. Rutishauser (1984) Guidance of optic axons in vivo by a preformed adhesive pathway on neuroepithelial endfeet. Dev. Biol. 106: 485-499.

Silver, J., and R. L. Sidman (1980) A mechanism for the guidance and topographic patterning of retinal ganglion cell axons. J. Comp. Neurol. 189: 101-111.

Singer, M., R. H. Nordlander, and M. Egar (1979) Axonal guidance during embryogenesis and regeneration in the spinal cord of the newt: The blueprint hypothesis of neuronal pathway patterning. J. Comp. Neurol. 185: 1-22.

Steinberg, M. S. (1970) Does differential adhesion govern self-assembly processes in histogenesis? Equilibrium configurations and the emergence of a hierarchy among populations of embryonic cells. J. Exp. Zool. 173: 395-434.

Tosney, K. W., and L. T. Landmesser (1984) Pattern and specificity of axonal outgrowth following varying degrees of chick limb bud ablation. J. Neurosci. 4: 2518-2527.

Wessells, N. K., P. C. Letourneau, R. P. Nuttall, M. Luduena-Anderson, and J. M. Geiduschek (1980) Responses to cell contacts between growth cones, neurites and ganglionic non-neuronal cells. J. Neurocytol. 9: 647-664 